\title{
DESCRIPCIÓN DE DOS ESPECIES NUEVAS DE LIOCALLONIA Y \\ TETRENTODON (GASTROPODA: PULMONATA: UROCOPTIDAE) PROCEDENTES DE LA COLECCIÓN "MIGUEL L. JAUME" DEL MUSEO NACIONAL DE HISTORIA NATURAL DE CUBA
}

\author{
Jane Herrera-Uria ${ }^{1}$ y José Espinosa ${ }^{2}$ \\ ${ }^{1}$ Museo Nacional de Historia Natural de Cuba. Obispo \#61 entre Oficios y Baratillo, Habana Vieja, \\ La Habana, Cuba. janehu@mnhnc.inf.cu \\ ${ }^{2}$ Instituto de Oceanología. Avda. $1^{a}$ \#18406 entre 184 y 186, Playa, \\ La Habana, Cuba. espinosa@oceano.inf.cu
}

\begin{abstract}
RESUMEN
Se describen dos especies nuevas de los géneros Liocallonia Pilsbry, 1902 y Tetrentodon Pilsbry, 1903, pertenecientes a la colección malacológica histórica "Miguel L. Jaume" depositada en el Museo Nacional de Historia Natural de Cuba. Se presentan fotografías de las conchas.
\end{abstract}

Palabras clave: Liocallonia, Tetrentodon, especies nuevas, Miguel L. Jaume, Cuba.

Title: Description of two new species of Liocallonia and Tetrentodon (Gastropoda, Pulmonata, Urocoptidae) from "Miguel L. Jaume" collection of the Cuban Museum of Natural History.

\section{ABSTRACT}

Two new species of the genera Liocallonia Pilsbry, 1902 and Tetrentodon Pilsbry, 1903 are described. These species belong to the "Miguel L. Jaume" historical malacological collection housed in the National Museum of Natural History of Cuba. Photographs of the shells are presented.

Keywords: Liocallonia, Tetrentodon, new species, Miguel L. Jaume, Cuba.

\section{INTRODUCCIÓN}

Miguel Luis Jaume es considerado como uno de los más ilustres malacólogos cubanos del pasado siglo XX. Comenzó a formar su colección privada de moluscos desde la década del 20 del pasado siglo, la cual pasó a formar parte del Museo Cubano de Ciencias Naturales desde el mismo año de su fundación. En 1989, la colección malacológica de Jaume fue dividida en tres; una parte quedó en el actual Museo Nacional de Historia Natural de Cuba y las otras dos fueron trasladadas al Instituto de Ecología y Sistemática de La Habana y al Museo "Jorge Ramón Cuevas" en la Reserva de Biosfera Baconao, Santiago de Cuba.

Como resultado de la rica y amplia obra científica de este malacólogo, a modo de resumen podemos decir que fue autor de más de 135 publicaciones, describió 752 moluscos nuevos para la ciencia, y le fueron dedicados más de 50 táxones entre géneros y especies. Una reciente revisión de su colección malacológica histórica "Miguel L. Jaume" depositada en el Museo Nacional de Historia Natural de Cuba (Herrera-Uria y Espinosa, 2015), reveló la presencia de dos especies nuevas de gasterópodos terrestres cubanos. Su diversa y extensa colección de moluscos, que aún en nuestros días al ser objeto de estudios, consultas y referencia, ofrece resultados tan sorprendentes como este. 
La familia Urocoptidae Pilsbry, 1898 es la más diversa de los moluscos terrestres cubanos, con más de 583 especies descritas, ordenadas en 4 subfamilias, 48 géneros y 71 subgéneros, la gran mayoría de ellos endémicos de Cuba. El género Liocallonia Pilsbry, 1902 (subfamilia Urocoptinae) abarca un total de 34 especies y 15 subespecies, incluyendo las subespecies nominales, ordenadas en tres subgéneros: Liocallonia (14 especies y 6 subespecies), Jaumea Jaume y Torre, 1976 (una especie) y Cenocoptis Jaume y Torre, 1976 (19 especies y 9 subespecies), véase Torre y Bartsch (2008); Espinosa y Ortea (2009). Mientras que el género Tetrentodon Pilsbry, 1903 (subfamilia Tetrentodoninae Bartsch, 1943) abarca un total de 78 especies y 8 subespecies, incluyendo las subespecies nominales, ordenadas en cinco subgéneros: Tetrentodon (58 especies), Anafecoptis Jaume y Torre, 1976 (una especie), Cylindrocoptis Jaume y Torre, 1976 (12 especies y 2 subespecies), Gravicoptis Jaume y Torre, 1976 (3 especies y 6 subespecies) y Scalaricoptis Jaume y Torre, 1976 (4 especies).

\title{
OBJETIVO
}

-Describir dos especies nuevas para la ciencia, correspondientes a los géneros Liocallonia Pilsbry, 1902 y Tetrentodon Pilsbry, 1903, provenientes de la colección malacológica histórica "Miguel L. Jaume", depositada en el Museo Nacional de Historia Natural de Cuba.

\section{MATERIALES Y MÉTODOS}

El material estudiado provino de la colección malacológica histórica "Miguel L. Jaume" depositada en el Museo Nacional de Historia Natural de Cuba (MNHNC). La identificación de las especies se realizó con la ayuda de un estereoscopio y las fotografías de las conchas fueron tomadas por los autores. En el ordenamiento taxonómico supragenérico se han seguido los criterios de Bouchet y Rocroi (2005) y las consideraciones expuestas por Espinosa y Ortea (2009) con relación a la autoría de los taxones genéricos y específicos de la familia Urocoptidae en Cuba. Siguiendo a Ortea $(1972,2014)$ se utiliza el Índice de desarrollo $(I d)$ dado por el cociente entre la longitud $(L)$ y la anchura de la concha $(A)$, de forma que $I d=L / A$. De acuerdo con dicho índice, las conchas serían muy anchas cuando su Id es menor de 2; anchas entre 2 y 2.4 , estrechas entre 2.4 y 2.8 y muy estrechas si es más de 2.8. Dicho $I d$ debe ser entendido como un caracter más, establecido a partir del material tipo.

\section{RESULTADOS Y DISCUSIÓN}

\author{
TAXONOMÍA \\ Familia Urocoptidae \\ Subfamilia Urocoptinae Pilsbry, 1902 \\ Género Liocallonia Pilsbry, 1902 \\ Subgénero Cenocoptis Jaume y Torre, 1976 \\ Liocallonia (Cenocoptis) torrebartschi sp. nov. \\ Figura 1, A-B
}

Diagnosis. Molusco de pequeño tamaño y fusiforme. Columela provista de tres lamelas, de las cuales la posterior es la más desarrollada y las otras dos son consecutivamente más pequeñas. Escultura axial bien desarrollada, formada por lamelas muy estrechas, bajas y retroactivas, bien separadas entre sí, algo irregulares en su disposición y desarrollo. 

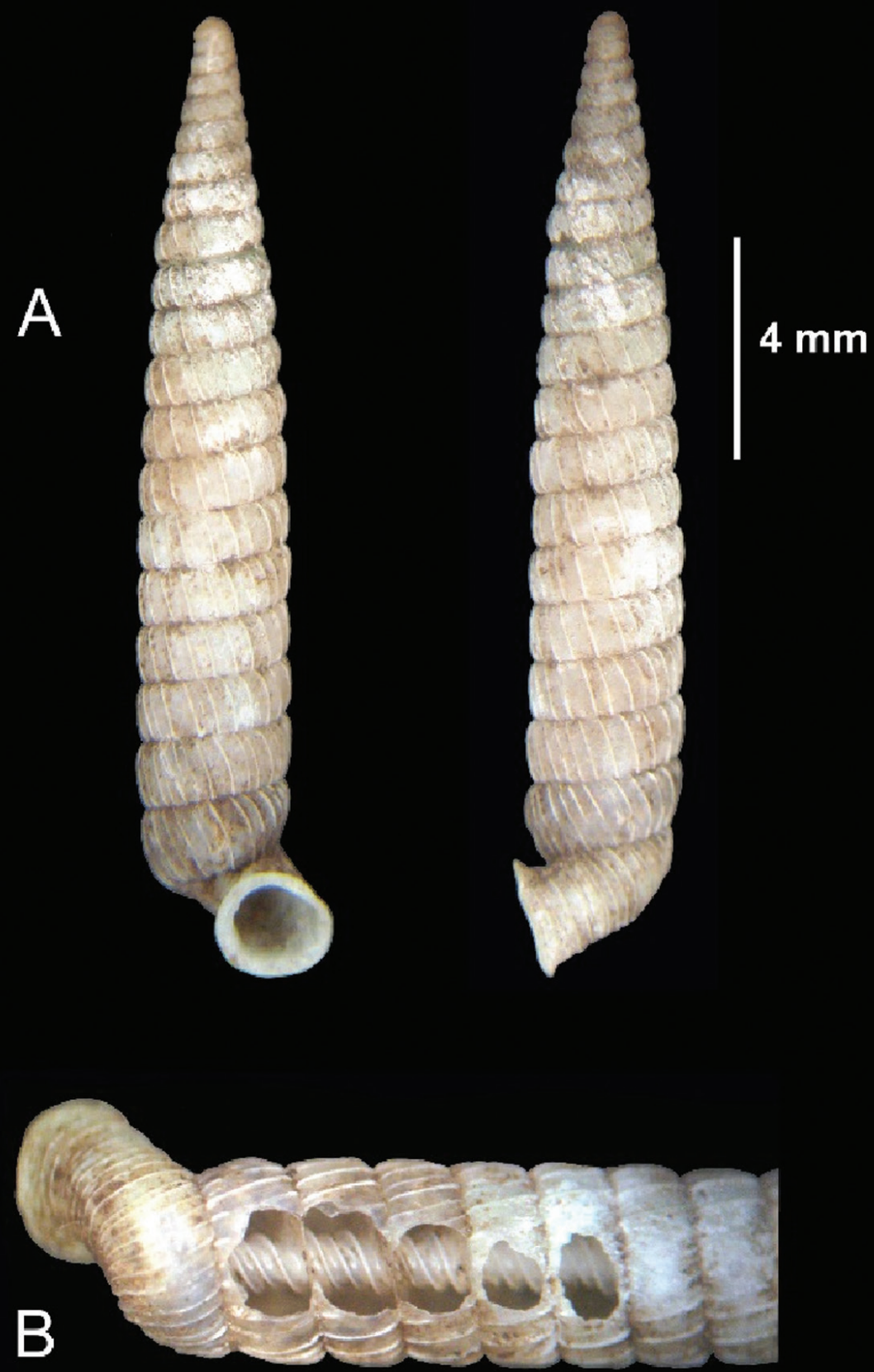

Figura 1. Liocallonia (Cenocoptis) torrebartschi, especie nueva. Holotipo (16.85 x $2.5 \mathrm{~mm})$. A, vista frontal y lateral de la concha. $\mathrm{B}$, vista de la columela. 
Diagnosis. A small, fusiform mollusc. Columella provided with three lamellae, the posterior one being the largest and the other two consecutively smaller. Axial sculpture well developed, formed by very narrow, low, and retroactive lamellae, widely discontinuous and somewhat irregular in their disposition and development.

Descripción del holotipo. Concha de forma turriculada alargada y muy estrecha $(\mathrm{Id}=6.74)$. Protoconcha formada por dos vueltas grandes y redondeadas, con la superficie cubierta por gránulos microscópicos muy pequeños. Teleoconcha con 18 vueltas moderadamente convexas y de perfil casi recto, separadas por una sutura relativamente fina y profunda, adornadas por numerosas costillas axiales lamelosas, muy estrechas, bajas y retroactivas, bien separadas entre sí, algo irregulares en su disposición y desarrollo. La última vuelta es adnata en su último un octavo final y presenta unas 26 lamelas axiales. En la base de la concha hay una seudofasciolasifonal con la escultura axial confluente en su interior. La abertura es subcircular, con el peristoma ligeramente reflejado y poco engrosado. Columela con tres lamelas espirales, la anterior más desarrollada que las otras dos, sin dentículos ni espinas. Color blanco sucio o gris claro, con la protoconcha pardo claro, mientras que el peristoma y el interior de la abertura son blancos.

Tipos. Un ejemplar de Los Mogotes del Manantial (localidad tipo), Vega del Palmar, Viñales, Pinar del Río, Cuba, identificado en la colección malacológica histórica "Miguel L. Jaume" del MNHNC como Cochlodinella sp. Holotipo (16.85 $\mathrm{mm}$ de largo y $2.5 \mathrm{~mm}$ de ancho máximo sin el peristoma) depositado en la colección básica de malacología del MNHNC, La Habana (MNHNCu-08.000161).

Etimología. Nombre en honor de los doctores Carlos de la Torre y Paul Bartsch por su significativo aporte al conocimiento de los moluscos terrestres cubanos y en particular a la familia Urocoptidae.

Comentario. Torre y Bartsch (2008) describen tres especies del subgénero Cenocoptis con costillas axiales lamelosas: Liocallonia (Cenocoptis) jaguanensis (15.4 x $3.4 \mathrm{~mm}$ ), de la Jaula de Azcui, Pinar del Río, Liocallonia (Cenocoptis) itineris (16.4 x $4.1 \mathrm{~mm}$ ), del Mogote Galalón, Pinar del Río, y Liocallonia (Cenocoptis) antoniensis (14.6 x $2.9 \mathrm{~mm})$, del Hoyo, Mogote La Laguna, San Antonio, Pinar del Río, las dos primeras de forma fusiforme comparativamente ancha $(\mathrm{Id}=4.52$ e $\mathrm{Id}=4.0$, respectivamente). Liocallonia antoniensis es más estrecha y alargada que las otras dos $(\mathrm{Id}=5.03)$, pero proporcionalmente es más ancha y menos alargada que L. torrebartschi, especie nueva, la que en adición tiene la porción final de la última vuelta más desprendida y la seudofasciolasifonal más marcada.

\section{Familia Urocoptidae}

Subfamilia Tetrentodoninae Barstch, 1943

Género Tetrentodon Pilsbry, 1903

Subgénero Cylindrocoptis Jaume y Torre, 1976

Tetrentodon (Cylindrocoptis) jaumei sp. nov.

Figura 2, A-B

Diagnosis. Molusco de pequeño tamaño y forma cilíndrica. Columela con un pliegue simple. Primeras vueltas, después de la decolación, no muy hinchadas. Teleoconcha adornada con costillitas axiales muy débiles, bajas y separadas entre sí, visibles en casi todas las vueltas centrales de la teleoconcha. 


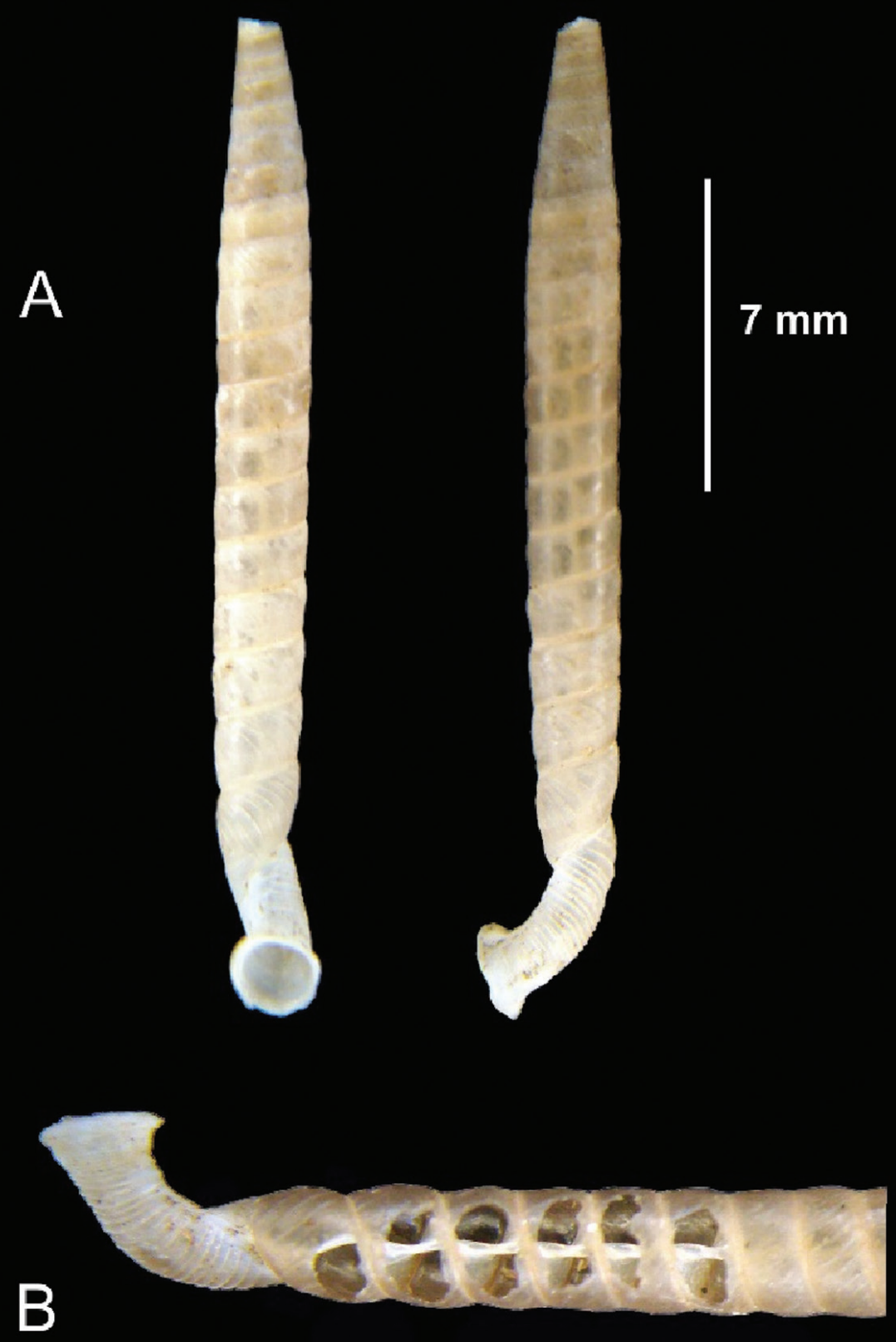

Figura 2. Tetrentodon (Cylindrocoptis) jaumei, especie nueva. Holotipo $(21.0$ x $2.04 \mathrm{~mm})$. A, vista frontal y lateral de la concha. $\mathrm{B}$, vista de la columela. 
Diagnosis. A small, cylindric mollusc. Columella with a simple twist. First whorls after decollation not greatly swollen. The whorls are marked by very weak, low, and discontinuous minute axial ribs, evident on most of the central part of the shell.

Descripción del holotipo. Concha de aspecto delicado, poco engrosada y algo translúcida, de forma subcilíndrica alargada y muy estrecha $(\mathrm{Id}=10.29)$. Protoconcha formada por unas dos vueltas grandes y redondeadas, con un núcleo bien marcado y la superficie cubierta por gránulos microscópicos muy pequeños. Teleoconcha con 19 vueltas moderadamente convexas, separadas por una sutura relativamente fina y profunda, adornadas por costillitas axiales muy débiles, bajas y separadas entre sí, visibles en casi todas las vueltas centrales de la teleoconcha y en las dos últimas vueltas las costillitas axiales se hacen más evidentes y numerosas. La última vuelta es casi completamente adnata. Columela interior con un pliegue simple. Color crema claro o pardo amarillento muy pálido, con las dos últimas vueltas de la teleoconcha más pálidas, casi blancas, mientras que la protoconcha y las primeras vueltas de la teleoconcha son de color pardo claro; el peristoma y el interior de la abertura son blancos.

Tipos. Once ejemplares recolectados en la Sierra del Grillo, puente al entronque de Aguacate (localidad tipo), Madruga, Mayabeque, Cuba, identificado en la colección malacológica histórica "Miguel L. Jaume" del MNHNC como Urocoptis menendezi (MNHNCu-88.428), nomennudum. Holotipo (21.0 mm de largo y $2.04 \mathrm{~mm}$ de ancho máximo sin el peristoma) depositado en la colección básica de malacología del MNHNC, La Habana (MNHNCu-08.000162).

Etimología. Nombre en honor al gran malacólogo Miguel Luis Jaume García, destacado naturalista cubano, cuyos aportes científicos, biblioteca y colección de moluscos constituyen fuentes de obligadas referencias y consultas en el estudio de este grupo zoológico en Cuba.

Comentario. Por su última vuelta soluta en casi su totalidad y la presencia de débil escultura axial en las vueltas centrales de la teleoconcha, Tetrentodon (Cylindrocoptis) jaumei, especie nueva puede ser discutida con Tetrentodon (Cylindrocoptis) striosa Torre y Bartsch, 2008 (21.4 x 1.9 $\mathrm{mm}$, sin el peristoma), de Ganuza, provincia Mayabeque, la cual se caracteriza por su forma más alargada y estrecha (29.3 vueltas de teleoconcha; $\mathrm{Id}=11.26)$, con las vueltas centrales de la teleoconcha más convexas y redondeadas y por su abertura subtriangular, entre otros caracteres morfológicos de la concha (véase Torre y Bartsch, 2008). Tetrentodon (Cylindrocoptis) gracillima (Poey, 1853), también con la última vuelta casi completamente soluta, y con dos subespecies descritas, T. gracillima gracillima, $(21.2 \times 1.9 \mathrm{~mm}$, sin el peristoma), de San José de Las Lajas, Mayabeque, y T. gracillima callida (16.6 x $1.8 \mathrm{~mm}$, sin el peristoma), de la Loma Candela, Mayabeque, se caracteriza por la ausencia de escultura axial visible en la porción media de las vueltas de la teleoconcha en ambas subespecies.

\section{AGRADECIMIENTOS}

Queremos agradecer especialmente a la Fundación Rufford (Ref. 19062-2) y a nuestro muy estimado Gilberto Silva Taboada por la información facilitada acerca de Miguel L. Jaume y sus muy oportunos comentarios. 


\section{LITERATURA CITADA}

Bouchet, P. y J. P. Rocroi. 2005. Classification and nomenclator of gastropod families. Malacologia, 47 (1-2): 1-397.

Espinosa, J. y J. Ortea. 2009. Moluscos terrestres de Cuba. Vaasa, Finland. 191 pp.

Herrera-Uria, J. y J. Espinosa. 2015. Los Tipos primarios de la colección malacológica histórica "Miguel L. Jaume", depositados en el Museo Nacional de Historia Natural de Cuba. Solenodon, 12: 151-160.

Ortea, J. 1972. Taxonomía numérica de Bivalvos. III European Philips Contest. Mimeografiado. Ediciones Dayton, Madrid. 42 pp.

Ortea, J. 2014. Cómo integrar ciencia y naturaleza: descripción de nuevas especies de Volvarina Hinds, 1844 (Mollusca: Marginellidae) de la isla de Guadeloupe y sus islotes satélites (Antillas Menores, Mar Caribe), nombradas en honor de treinta mujeres distinguidas con el premio 1'Oreal-UNESCO. Revista de la Academia Canaria de Ciencias, Vol. XXVI: 129-188.

Torre, C. de la y P. Bartsch. 2008. Los moluscos terrestres cubanos de la familia Urocoptidae. Editorial Científico-Técnica. 800 pp.

[Recibido: 16 de febrero, 2016. Aceptado para publicación: 25 de agosto, 2016] 